\title{
Elevation of HO-1 Expression Mitigates Intestinal Ischemia-Reperfusion Injury and Restores Tight Junction Function in a Rat Liver Transplantation Model
}

\author{
Xinjin Chi, ${ }^{1}$ Weifeng Yao, ${ }^{1}$ Hua Xia, ${ }^{2}$ Yi Jin, ${ }^{3} \mathrm{Xi} \mathrm{Li}^{4}{ }^{4} \mathrm{Jun} \mathrm{Cai},{ }^{1}$ and Ziqing Hei ${ }^{1}$ \\ ${ }^{1}$ Department of Anesthesiology, Third Affiliated Hospital, Sun Yat-Sen University, Guangzhou, Guangdong 510630, China \\ ${ }^{2}$ Department of Anesthesiology, The Affiliated Hospital of Luzhou Medical College, Luzhou, Sichuan 646000, China \\ ${ }^{3}$ Department of Pathology, Third Affiliated Hospital, Sun Yat-Sen University, Guangzhou, Guangdong 510630, China \\ ${ }^{4}$ Department of Breast and Thyroid Surgery, Third Affiliated Hospital, Sun Yat-Sen University, Guangzhou, Guangdong 510630, China
}

Correspondence should be addressed to Xinjin Chi; chixinjin@yeah.net and Ziqing Hei; heiziqing@sina.com

Received 11 January 2015; Accepted 26 April 2015

Academic Editor: Tilman Grune

Copyright (C) 2015 Xinjin Chi et al. This is an open access article distributed under the Creative Commons Attribution License, which permits unrestricted use, distribution, and reproduction in any medium, provided the original work is properly cited.

\begin{abstract}
Aims. This study was aimed at investigating whether elevation of heme oxygenase-1 (HO-1) expression could lead to restoring intestinal tight junction (TJ) function in a rat liver transplantation model. Methods. Intestinal mucosa injury was induced by orthotopic autologous liver transplantation (OALT) on male Sprague-Dawley rats. Hemin (a potent HO-1 activator) and zincprotoporphyrin (ZnPP, a HO-1 competitive inhibitor), were separately administered in selected groups before OALT. The serum and intestinal mucosa samples were collected at 8 hours after the operation for analysis. Results. Hemin pretreatment significantly reduced the inflammation and oxidative stress in the mucosal tissue after OALT by elevating HO-1 protein expression, while $\mathrm{ZnPP}$ pretreatment aggravated the OALT mucosa injury. Meanwhile, the restriction on the expression of tight junction proteins zonula occludens- 1 and occludin was removed after hemin pretreatment. These molecular events led to significant improvement on intestinal barrier function, which was proved to be through increasing nuclear translocation of nuclear factor-E2-related factor 2 (Nrf2) and reducing nuclear translocation of nuclear factor kappa-B (NF- $\kappa \mathrm{B})$ in intestinal injured mucosa. Summary. Our study demonstrated that elevation of HO-1 expression reduced the OALT-induced intestinal mucosa injury and TJ dysfunction. The HO-1 protective function was likely mediated through its effects of anti-inflammation and antioxidative stress.
\end{abstract}

\section{Introduction}

For more than 30 years liver transplantation has been an effective treatment procedure for end-stage liver diseases [1, 2]. One- and five-year survival rate following liver transplantation are $90 \%$ and $75 \%$, respectively [3]. One of challenges during liver transplantation is the organ injury. Liver is particularly susceptible to ischemia-reperfusion injury as a result of storage, transportation, and operational procedure from donor to recipient [4]. Indeed, acute organ injury leads to poor early recovery in recipients. Organ injury is not limited to the liver. We have demonstrated that the intestine injury occurred in a rat orthotopic autologous liver transplantation (OALT) model [5]. Intestine injury can lead to the spread of intraluminal bacteria and endotoxin to organs and tissues beyond the intestine. Diffusion of bacteria and toxins is a primary cause of systemic inflammatory response syndrome (SIRS), MODS, and multiple organ failure $[6,7]$.

During liver transplantation procedure, the recipients inevitably experienced a short anhepatic phase (including occlusion of portal vein that leads to intestine congestive ischemia) and gastrointestinal congestion for about 45$65 \mathrm{~min}$ or longer [8]. The epithelial cells of the intestinal mucosa are sensitive to the hypoxia-ischemia upon intestinal congestion, and they are often subjected to apoptosis and necrosis after the surgery [9]. Meanwhile, the transplanted liver also inevitably underwent ischemia-reperfusion injury during the process. These factors are causatively associated with postoperative complications including the damage to intestinal barrier and the barrier dysfunction, and the increase of the postoperative mortality rate. However, 
the mechanisms underlying intestinal barrier dysfunction during the perioperative period remain much unknown.

Tight junction (TJ) refers to the closely shared areas between two neighboring cells where membranes at both sides join together to form a virtually impermeable barrier. Compromised TJ barrier structure increases paracellular permeability. The resulting release of proinflammatory molecules within GI track could trigger mucosal immune system activation, leading to tissue damage consisting of ongoing inflammatory reaction and oxidative stress. Evidence from clinical and experimental studies indicated that alteration of intestinal TJ barrier is critical in leading to intestinal dysfunction [10-12].

Heme oxygenase $(\mathrm{HO})$ is an enzyme specialized in degrading heme and is assembled with biliverdin, CO [13], and free iron [14]. HO-1 is normally expressed in the mucosal layer of gastrointestinal track $[15,16]$. HO-1 expression is upregulated rapidly to respond to stress and injury in the mucosa [17]. Indeed, many studies have shown that HO-1 exerted protective functions against stress-related tissue damage [17-19]. The upregulation of HO-1 upon stress suggests that $\mathrm{HO}-1$ is a part of intrinsic network to mitigate tissue injury in the gastrointestinal tract [20].

Upon intestine injury detection of metabolic products released by overgrown bacteria is useful for diagnosing the intestinal barrier disorder. D-Lactate (D-LA) is a product released by many of microflora resided in the human gastrointestinal tract. Thus increase of serum D-LA level is a sign of intestinal ischemia [21-23]. Diamine oxidase (DAO) is only synthesized in epithelial cells of intestinal villi which are usually an epicenter of the ischemia. Released DAO by damaged mucosal cells will increase its serum concentration. Intestinal-type fatty acid-binding protein (FABP2) is exclusively released by the damaged intestinal epithelial cells [24]. Intestinal mucosal barrier is a protective complex structure primarily containing tight junctions (TJs); TJs seal individual mucosal membranes at conjunction. The integrity of TJs is the foundation of the structural stability and functional effectiveness of the epithelial barrier $[25,26]$. Occludin, tricellulin, claudin, and functional adhesion molecules are internal component of TJs structure [27-29]. It is expected to detect altered levels of those proteins in both serum and injured epithelial cells upon OALT ischemia and other stress [30].

We hypothesized that HO-1 expression was significantly elevated to respond to OALT-induced organ injury. The current study was aimed at investigating whether elevation of HO-1 expression could restore intestinal barriers function through protecting TJ structure and if anti-inflammation and antioxidative stress pathway was the mechanistic contributor to generating protective function in a rat liver transplantation model.

\section{Methods and Materials}

2.1. Animals. Male Sprague-Dawley rats (weighing 200$250 \mathrm{~g}$ ) were cohosted in individual cages in a temperaturecontrolled room with a specific pathogen-free, laminar flow atmosphere in the Department of Laboratory Animal Center at Sun Yat-Sen University. The animal room was switched to dark after $12 \mathrm{hr}$ light each day. They were given a week to get accustomed to environment before the experiment. Rats were allowed to drink water, but were fasted for $8 \mathrm{hrs}$ prior to the study. Animal protocols were approved by the Sun YatSen University Animal Care Committee, and the experiments were performed with adherence to the guidelines provided by the National Institutes of Health for the use of animals in laboratory experiments.

\subsection{Orthotopic Autologous Liver Transplantation (OALT)} Model. Rats were sedated with intraperitoneal injection of pentobarbital $(30 \mathrm{mg} / \mathrm{kg}$ ) under $50 \%$ oxygen delivered using an animal mask. A standard OALT model was created as previously published $[31,32]$. The entire operation procedures were aseptic. The model simulates the main hemodynamic processes during perioperative period of liver transplantation, in which the anhepatic phase can be controlled without inducing the complications of rejection or other nonsurgical factors in orthotopic liver transplantation using the cuff technique.

2.3. Hemin and ZnPP. Hemin and ZnPP (Sigma-Aldrich, St. Louis, MO) were solubilized in diluted solution containing sodium hydroxide $(0.1 \mathrm{~mol} / \mathrm{L})$; hydrochloride acid was used to lower the $\mathrm{pH}$ to 7.4 and then diluted to $5 \mathrm{mg} / \mathrm{mL}$ (Hemin) and $4 \mathrm{mg} / \mathrm{mL}(\mathrm{ZnPP})$ using $0.85 \%$ saline solution, respectively.

2.4. Animals Groups. Six experimental animal groups $(n=$ 3-6/group) were established with random animal selection: sham + saline, sham + hemin and sham + ZnPP, OALT + saline, OALT + hemin, and OALT + ZnPP. Rats were intraperitoneally injected with saline, hemin $(30 \mathrm{mg} / \mathrm{kg})$, and $\mathrm{ZnPP}(20 \mathrm{mg} / \mathrm{kg}$ ) separately in designated groups $24 \mathrm{~h}$ before operation and then received celiotomy and vascular separation with or without OALT at 8 hours after OALT. We assumed no significant discrepancy between two sham groups with respective $\mathrm{ZnPP}$ and hemin pretreatment; the results were pooled from two groups (sham + hemin) and $($ sham $+\mathrm{ZnPP})$ and were merged as a single sham group.

2.5. Collection of Intestinal Mucosa. The animals were sacrificed with an overdose of pentobarbital $(200 \mathrm{mg} / \mathrm{kg}$ i.p. $)$ at $8 \mathrm{~h}$ after OALT. We carefully removed the entire small intestine and cut a $0.5 \mathrm{~cm}$ length of intestine that was approximately $10 \mathrm{~cm}$ distance from the end of ileum. We fixed the tissue in $4 \%$ (concentration) formalin diluted in PBS and then paraffin-embedded it for section. We completely washed the remaining small intestine with $0.9 \%$ sodium chloride that was precooled at $4^{\circ} \mathrm{C}$ and then exposed the intestinal epithelium by cutting one side of the wall linearly. We rinsed the opened epithelium with precooled $0.9 \%$ sodium chloride and dried it by blotting off the remaining moisture with filter paper. We harvested the mucosal epithelium by gentle separation of it from the wall with a glass slide within a plate on the ice and then it was stored at $-80^{\circ} \mathrm{C}$ for further analysis [33]. 
2.6. Histological Examination. Sections at five $\mu \mathrm{m}$ thickness were cut from the blocks described above and stained with hematoxylin-eosin (H\&E) for viewing histologic changes. Two pathologists who were blind to coded samples scored the mucosal inflammation and injury and average core for each sample was used for comparison [33].

\subsection{Intestine Malonaldehyde (MDA) Concentration and Super-} oxide Dismutase (SOD) Activity. Intestinal mucosa was homogenized with normal saline. The MDA, a lipid peroxidation end product in the tissue with oxidative stress, was TBA assayed (Jiancheng Bioengineering Ltd., Nanjing, China). The MDA level in intestine was expressed as millimole/mg (protein). Once intestine tissue homogenate was ready, it was centrifuged for $15 \mathrm{~min}$ at $3000 \mathrm{~g}$ after a $5 \mathrm{~min}$ incubation at $-20^{\circ} \mathrm{C}$. The supernatant was separated from pellet and used for SOD activity detection by SOD detection kit (Jiancheng Bioengineering Ltd., Nanjing, China). The activity of SOD in intestinal mucosa was calculated as $\mathrm{U} / \mathrm{mg}$ (protein) [5].

2.8. Enzyme-Linked Immunosorbent Assay. D-Lactic acid (D-LA, Biosamite Biotechnology Co. Ltd., China), diamine oxidase (DAO), and intestinal-type fatty acid-binding protein (FABP2) (both from Cloud-Clone Corp., USA) in serum and IL- 6 and TNF- $\alpha$ levels in intestinal mucosa were measured following the standard ELISA procedure (Jiancheng Bioengineering Ltd., Nanjing, China).

2.9. Immunohistochemical Assay for Cleaved Caspase-3. The $5 \mu \mathrm{m}$ sections cut from the tissue blocks were subjected to standard procedure for dewaxing, blocking endogenous peroxidase and exposing antigenic sites before immunohistochemical staining. Mouse anti-cleaved caspase- 3 antibody (Santa Cruz, CA) was diluted at 1:200 as primary antibody. Positive signal was visualized with $3,3^{\prime}$-diaminobenzidine (DAB, Dako Cytromation, USA) color reaction. Nuclei were stained with hematoxylin. Under the code five fields per each slide at random choice of the viewer were semiquantified.

2.10. Immunofluorescence. Briefly, potential nonspecific staining in the sections was blocked with $5 \%$ bovine serum albumin and $0.3 \%$ Triton X-100 in PBS. Mouse anti-Nrf2 $(1: 100)$ (Abcam, UK) or anti-NF- $\kappa$ B (1:100) (Abcam, UK) antibodies were used as primary antibodies and then followed by a secondary antibody conjugated with fluorescence $(1: 100)$ (Life technologies, USA). Fluorescent microscope (Leica, DMLB2, Germany) was utilized for viewing the stained sections.

2.11. Western Blot Assay. We followed the standard protein separation and blotting procedures. Monoclonal antibody to zonula occludens-1 (ZO-1), occludin, $\mathrm{HO}-1$, and $\beta$-actin (Santa Cruz) were used as primary antibodies, and the HPconjugated anti mouse IgG (Cell Signaling Technology) as secondary antibody. Protein bands were detected by ECL kit (enhanced chemiluminescence detection KGP1125, Nanjing KeyGen Biotech. Co., Ltd.); $\beta$-actin band density was used as loaded sample reference to normalize relative level of each detected protein [32].

2.12. TUNEL Staining. Apoptosis in the intestine sections was examined after TUNEL staining with in situ cell death detection kit (Roche, Basel, Switzerland). The DAPI (Invitrogen) was used to stain nuclei. The average number of apoptotic cells was calculated from five random fields.

2.13. Statistical Analysis. Quantitative data are presented as mean \pm standard error. Each sample was analyzed in triplicate for all biochemical assays. Therefore, all the data were means of triplicate measurements. Significance was evaluated using one-way ANOVA test (SPSS 13.0, SPSS Inc., Chicago, III) followed by Tukey post hoc multiple comparisons test for unpaired values. Statistical significance was called when $p<$ 0.05 .

\section{Results}

3.1. Histopathological Analyses of Intestines in Animals with $O A L T$. In order to assess the histopathological changes induced with the treatment protocols, two pathologists independently scored the intestinal mucosa injury for each of coded samples and average score was used for analysis (Figure 1(b)). Results showed that a serious intestine injury occurred at $8 \mathrm{~h}$ after OALT ( $p<0.01$ versus sham). OALT procedure caused cytopathological changes featured with necrosis and inflammation in the intestine mucosa in group pretreated with saline. However, the intestinal injury score was sharply decreased in the animals pretreated with a HO-1 activator hemin in OALT + hemin group $(p<0.01$ versus OALT + saline). Further, the injury score, in the animals pretreated with $\mathrm{HO}-1$ inhibitor in OALT + ZnPP group, was elevated comparing the score in OALT + saline group $(p<$ 0.05 versus OALT + saline).

3.2. Restoration of Intestinal TJ Was Accompanied by Elevated HO-1 Expression. Since epithelial TJ regulates paracellular permeability the expression of ZO-1 and occludin, selected from TJ proteins, was analyzed by Western blotting (Figure 4). OALT reduced the intestinal ZO-1 and occludin expression, accompanied by an increase in HO-1 expression. Treatment with hemin at $24 \mathrm{~h}$ before operation significantly elevated HO-1 protein level comparing OALT-induced HO-1 increase. We investigated whether elevation of HO-1 expression can protect the intestinal cells against OALT-induced barrier dysfunction. OALT-induced reduction of ZO-1 and occludin were mitigated by the hemin pretreatment $(p<$ 0.05 versus OALT + saline). Conversely, after treatment with $\mathrm{ZnPP}$, the ZO-1 and occludin levels were lower than those in OALT + saline $(p<0.05)$.

3.3. The Elevation of HO-1 and Restoration of Intestinal Barrier Function after OALT. Concentrations of Dlactic acid (D-LA) (Figure 2(a)), diamine oxidase (DAO) (Figure 2(b)), and intestinal-type fatty acid-binding protein (FABP2) (Figure 2(c)) were used as biomarkers to assess the 

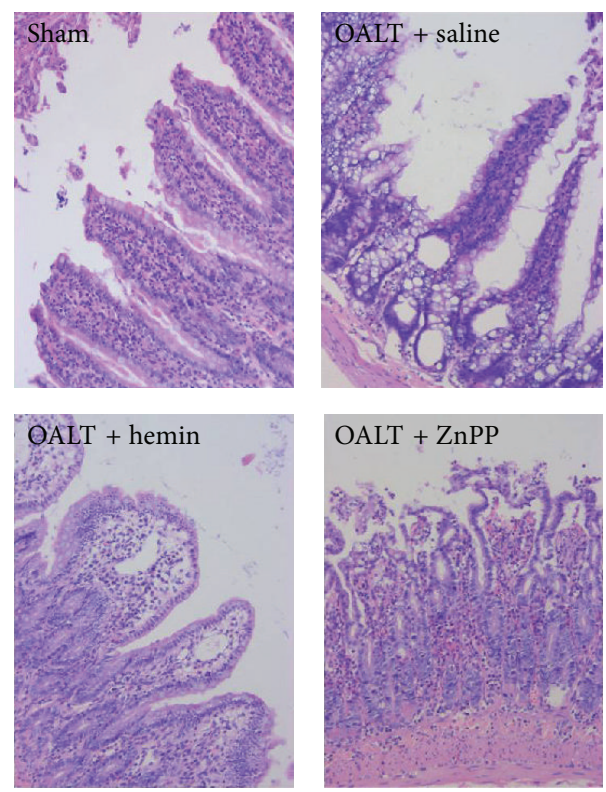

(a)

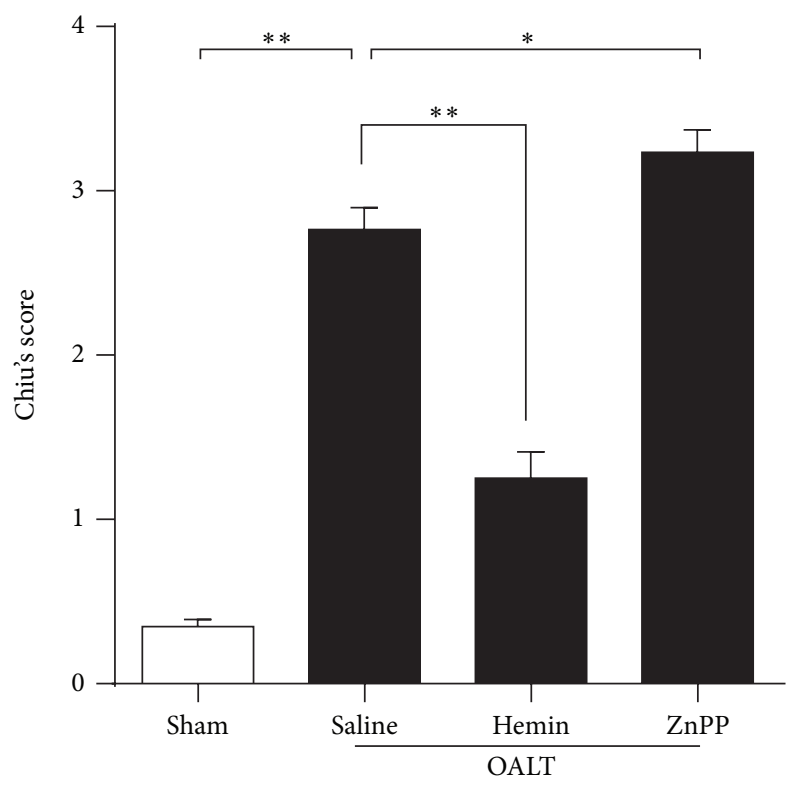

(b)

FIGURE 1: Histopathologic changes in intestines after orthotopic autologous liver transplantation (OALT). HE-stained intestine sections collected at $8 \mathrm{~h}$ after reperfusion from the sham, OALT + saline, OALT + hemin, and OALT + ZnPP groups (a) $(\times 200)$. Rats were intraperitoneally injected with saline, hemin $(30 \mathrm{mg} / \mathrm{kg})$, and ZnPP $(20 \mathrm{mg} / \mathrm{kg})$ separately in corresponding groups $24 \mathrm{~h}$ before operation and then received celiotomy and vascular separation with or without OALT. Intestinal mucosa injury was graded by Chiu's score (b). The data were presented as the mean $\pm \mathrm{SD}, n=3-6$ per group. ${ }^{*} p<0.05,{ }^{* *} p<0.01$, one-way ANOVA with Tukey test.

integrity of intestinal epithelia in this study. Significantly higher serum D-LA, DAO, and FABP2 levels were detected in OALT animals pretreated with saline than those without OALT ( $p<0.05$ versus sham). Statistically significant lower serum D-LA, DAO, and FABP2 levels were detected in the group that received $30 \mathrm{mg} / \mathrm{kg}$ of hemin $24 \mathrm{~h}$ before operation compared to OALT + saline animals $(p<0.05)$. As expected, the rats received $30 \mathrm{mg} / \mathrm{kg}$ of $\mathrm{ZnPP}$ showed higher levels of D-LA, DAO, and FABP2 than those in OALT + saline group $(p<0.05)$.

3.4. Elevation of HO-1 Expression Protected the Intestine Epithelial Cells from Apoptosis Caused by OALT. To further investigate apoptosis, the fragmented DNA and the cleaved caspase-3 expression were detected in the sections stained with TUNEL assay (Figure 3$)$. Hemin $(20 \mathrm{mg} / \mathrm{kg}$ ) pretreated regressed DNA fragmentation (Figures 3(a) and 3(c)) and reduced the cleaved caspase-3 expression level in comparison to group OALT + saline (Figures 3(b) and 3(d)), implying that OALT-induced apoptosis in the epithelia could be mitigated by HO-1 activation. Furthermore, ZnPP $(30 \mathrm{mg} / \mathrm{kg})$ aggravated the apoptosis in the epithelial cells after OALT, evidenced by significantly more fragmented DNA and cleaved caspase- 3 expression in intestine than that in OALT + saline group $(p<0.05)$.

3.5. The HO-1 Protective Function Was Related to the Activation of Transcription Factor Nrf2 and Reduction of NF- $\kappa B$. $\mathrm{Nrf2/ARE/HO}-1$ pathway is closely involved in protecting liver transplantation-induced remote organ injury. Sections from the sham group contained a few nuclear Nrf2-positive cells in epithelium lamina propria after immunofluorescent staining (Figures 5(a)-5(b)); the prominently increased nuclear Nrf2-positive cells in the saline intestinal tissue (epithelium and lamina propria) were detected, accompanied by elevation of the end product of lipid peroxidation malonaldehyde (MDA) (Figure 5(c)). The hemin treatment significantly activated the Nrf2 nuclear translocation in intestine epithelium and lamina propria and increased the activity of superoxide dismutase (SOD) of mucosa (Figure $5(\mathrm{~d})$ ) at $8 \mathrm{~h}$ after OALT. However, ZnPP treatment significantly inhibited the translocation of $\mathrm{Nrf} 2$.

Liver transplantation-induced remote organ injury is largely NF- $\kappa \mathrm{B}$ pathway dependent. The activation of NF$\kappa \mathrm{B}$ was also determined by immunofluorescent staining. The nuclear transportation of $\mathrm{NF}-\kappa \mathrm{B}$ in intestine lamina propria and its downstream cytokines IL-6 (Figure 5(g)) and TNF- $\alpha$ (Figure 5(h)) in intestinal tissue of the OALT + hemin group were markedly lower (Figure 5(e)) than those in the OALT group. More translocations were observed in OALT $+\mathrm{ZnPP}$ group than hemin group, suggesting that HO-1 activation inhibits chain inflammation reactions.

\section{Discussion}

Intestine injury associated with liver transplantation can result in bacterial or endotoxin translocation in both the experimental and clinical setting [34-36]. The physiopathological changes in liver transplantation-induced intestinal 


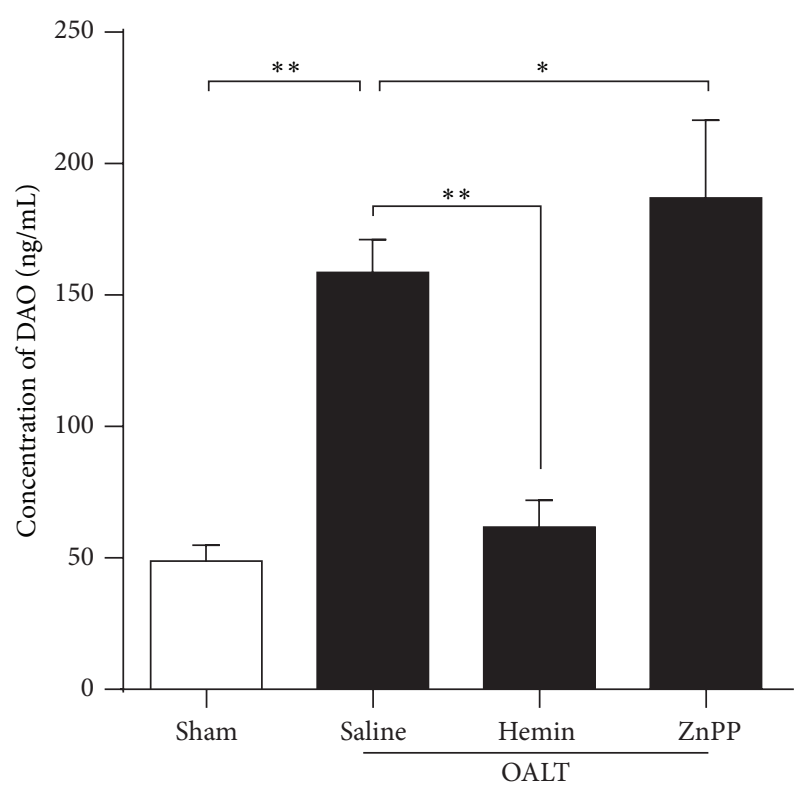

(a)

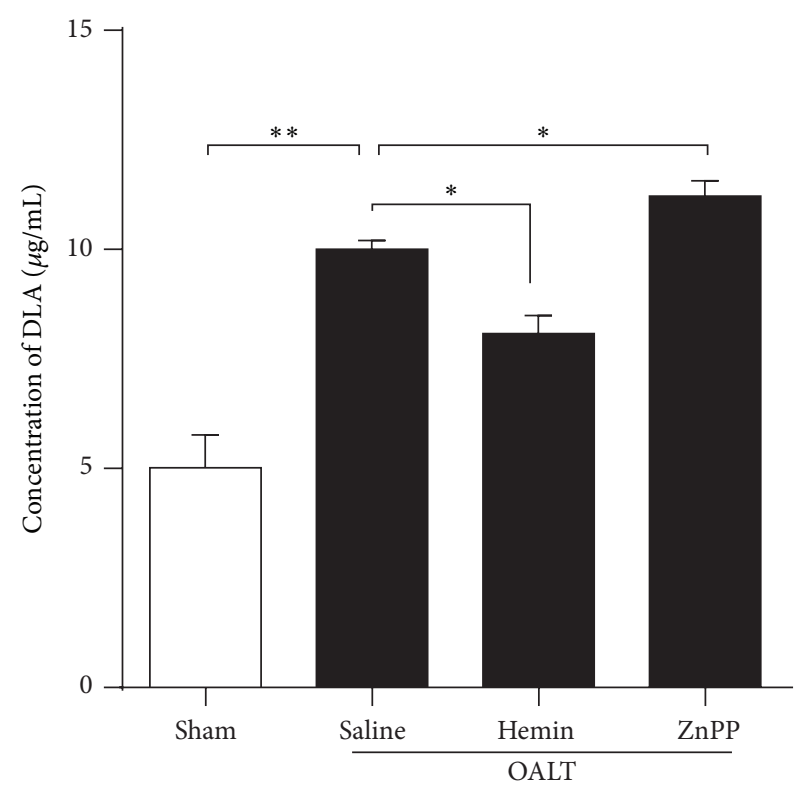

(b)

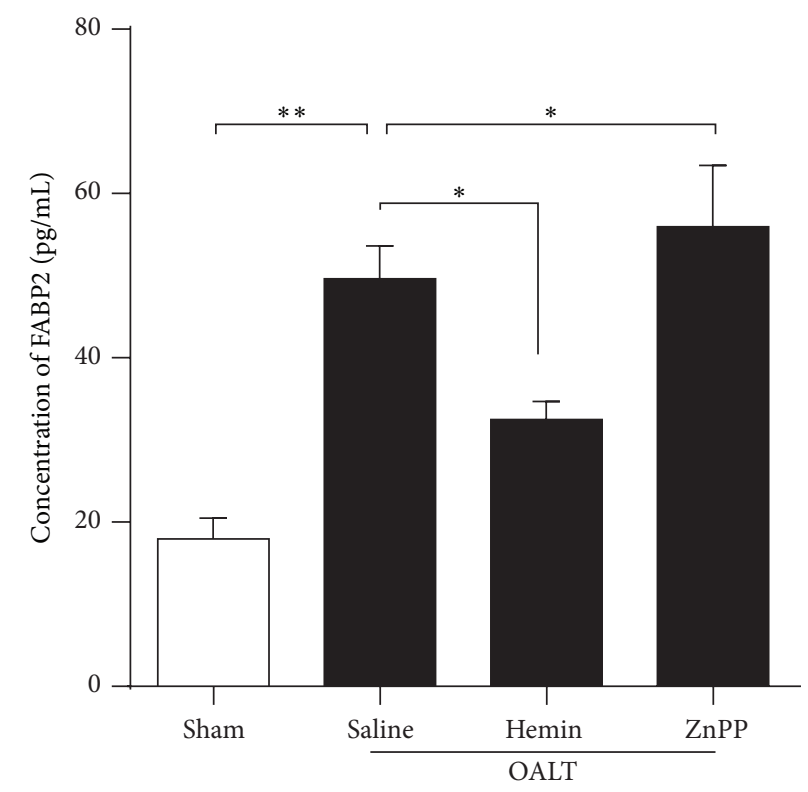

(c)

FIGURE 2: The impact of HO-1 expression level on intestinal barrier function after OALT. Concentrations of diamine oxidase (DAO) (a), Dlactic acid (D-LA) (b), and intestinal fatty acid-binding protein (FABP2) (c) in serum were detected to determine intestinal epithelial function. The serum was collected from each animal in all groups at $8 \mathrm{~h}$ after reperfusion. The results were presented as the mean \pm SD, $n=3-6$ per group. ${ }^{*} p<0.05,{ }^{* *} p<0.01$, one-way ANOVA with Tukey test.

injury include cytotoxicity and pathological changes in the intestinal mucosa barrier. Moreover, the intactness of gastrointestinal tract could have profound impact on functionality of other organs as GI has been called "motor of multiple organ failure." Such interdependence makes liver the most vulnerable organ after intestinal injury because liver and intestine share the anatomical common pathway such as coupled vasculature [37]. Restoration of intestine barrier function through protecting the barrier structure integrity may facilitate recipients recovering from liver transplantation procedure that causes systemic changes in physiopathology. However, the detailed mechanisms of intestinal barrier dysfunction induced by liver transplantation remain much unknown.

We investigated intestinal barrier dysfunction in orthotopic autologous liver transplantation (OALT) and the protection from injury by hemin-elevated HO-1 expression in this study. Severe intestinal mucosal injury appeared at $8 \mathrm{~h}$ after OALT. The falling of epithelial cells, breaking of villi, fusing of adjacent villi, mucosal atrophy, and edema were among 
TUNEL
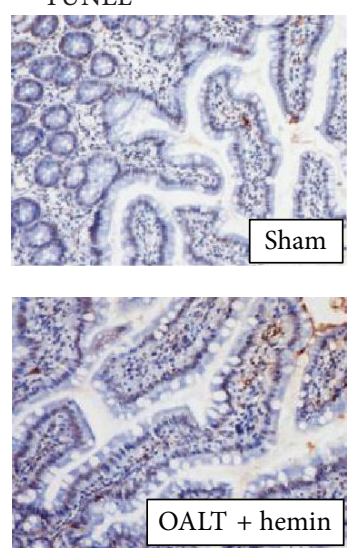

(a)

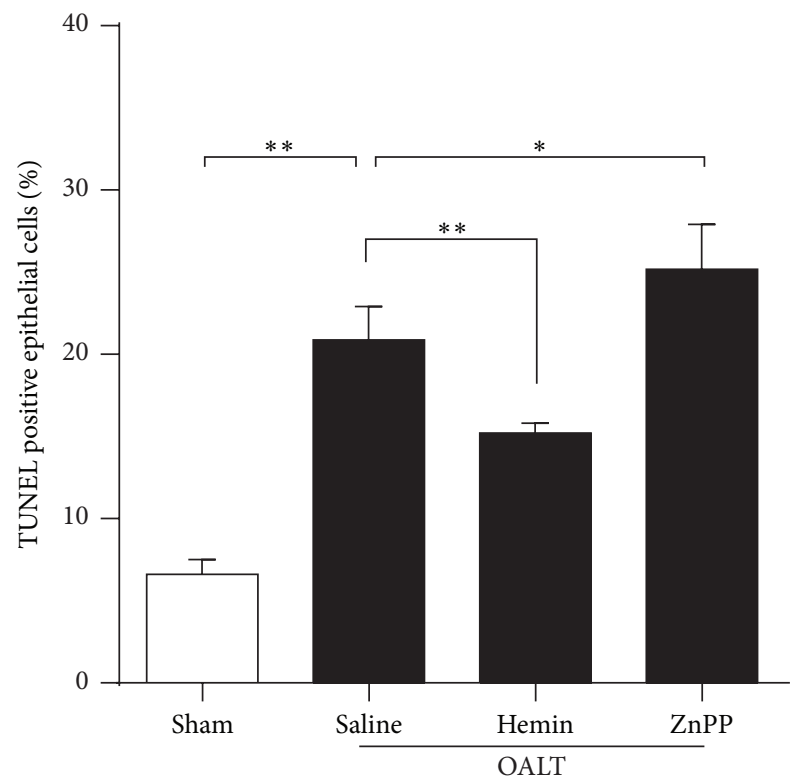

(c)
Cleaved caspase- 3
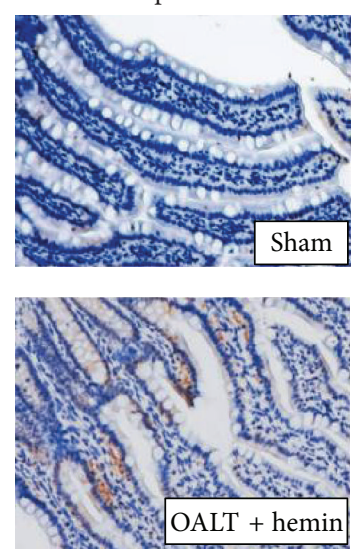

(b)

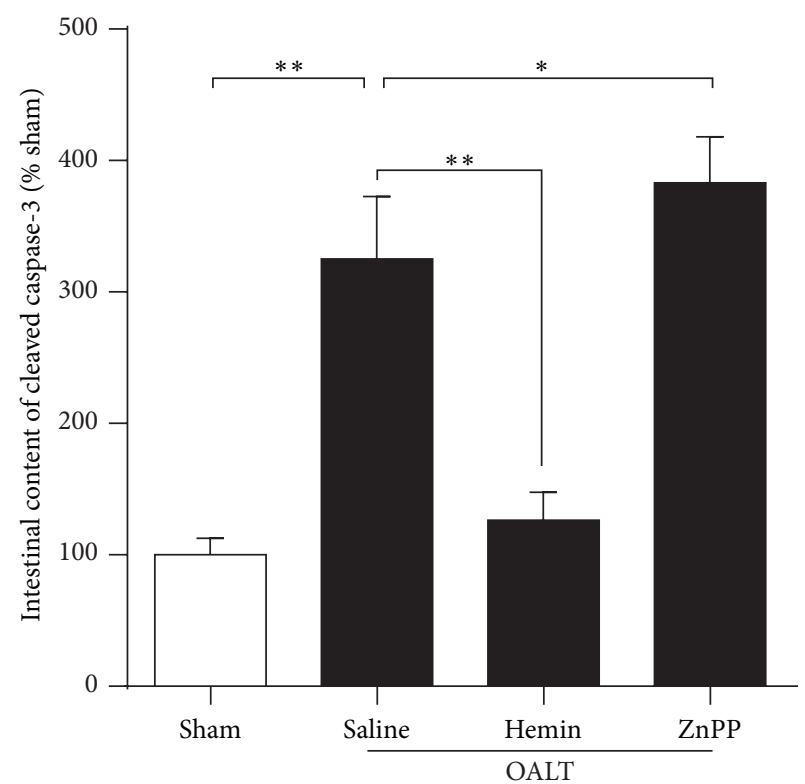

(d)

FIGURE 3: Elevation of HO-1 expression protected the intestine epithelial cells from apoptosis caused by OALT. Immunohistochemical staining of intestine sections in all 5 groups at $8 \mathrm{~h}$ after reperfusion for analysis of apoptosis using TUNEL assay (a); the expression of cleaved caspase- 3 (b); the number of apoptotic cells from TUNEL staining (c); the expression levels of cleaved caspase-3 from immunohistochemical staining (d). The results were expressed as the mean $\pm \mathrm{SD}, n=3$-6/group. ${ }^{*} p<0.05,{ }^{* *} p<0.01$, one-way ANOVA with Tukey test.

main pathological changes in the intestinal mucosa. Intestinal HO-1 protein expression level turned higher after OALT than that in the sham group, indicating that upregulation of HO-1 expression might represent an intrinsic protection against injury after OALT. These results were consistent with additional HO-1 upregulation induced by the pretreatment with hemin, resulting in the detectable mitigation of systemic inflammatory response and oxidative stress. HO-1 activation induced protective function was further supported by zincprotoporphyrin ( $\mathrm{ZnPP}$ ) treatments, which inhibited $\mathrm{HO}-1$ expression in the intestinal epithelia, leading to a severer intestinal mucosa injury after rats receiving OALT. All data suggest a protective function of HO-1 in the event of injury. Hemin has been widely used as a pharmacological agent to upregulate $\mathrm{HO}-1$ expression to mitigate injury upon inflammation [38]. The negative impact on $\mathrm{HO}-1$ protective property in presence of $\mathrm{HO}-1$ inhibitor $\mathrm{ZnPP}$ is in agreement with the published observations that the $\mathrm{ZnPP}$ inhibition of HO-1 function [39] abolished the HO-1 beneficial effects.

Next, we dissected the detailed mechanism underlying the intestine injury. The intestinal mucosa is highly susceptible to ischemia and hyperpermeability can result from the injury of mucosa. The structure of TJ and epithelial barrier is maintained essentially through the interaction between occludin and ZO-1 [40]. In this study, we detected significantly lower occludin and ZO-1 expression in intestinal epithelia at $8 \mathrm{~h}$ after OALT. The lower expression of occludin and $\mathrm{ZO}-1$ was accompanied by increasing concentrations of D-LA, DAO, and FABP2 in serum, which clearly suggested a compromised TJs structure leading to the epithelial barrier 


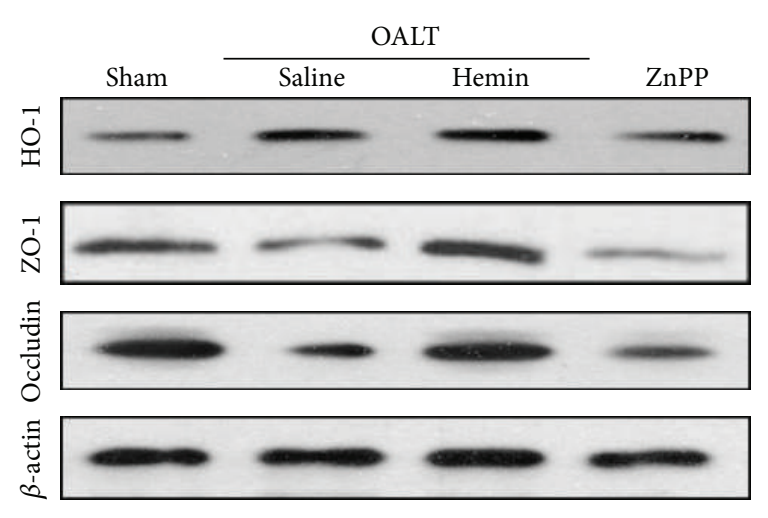

(a)

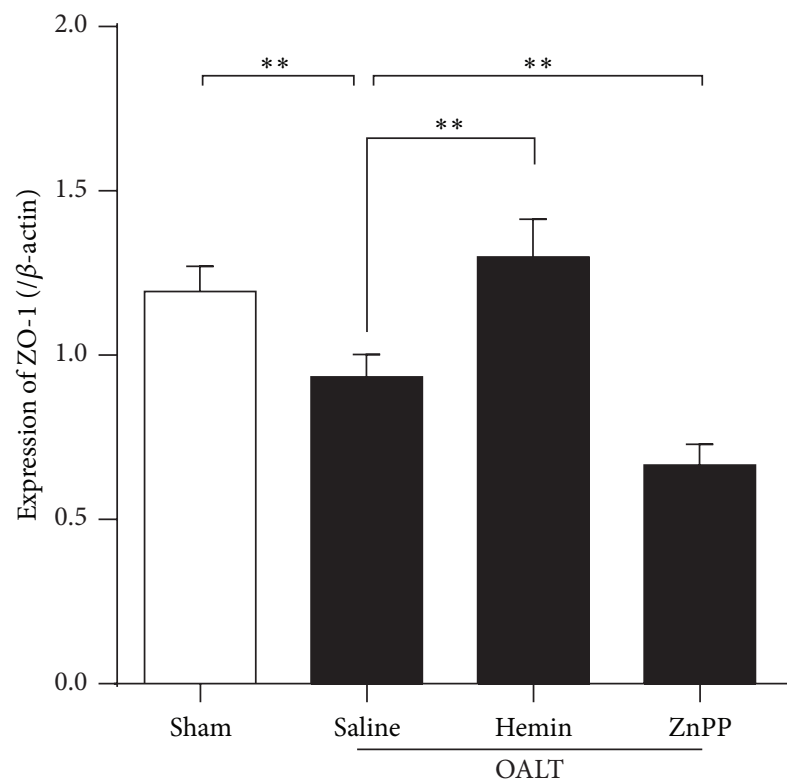

(c)

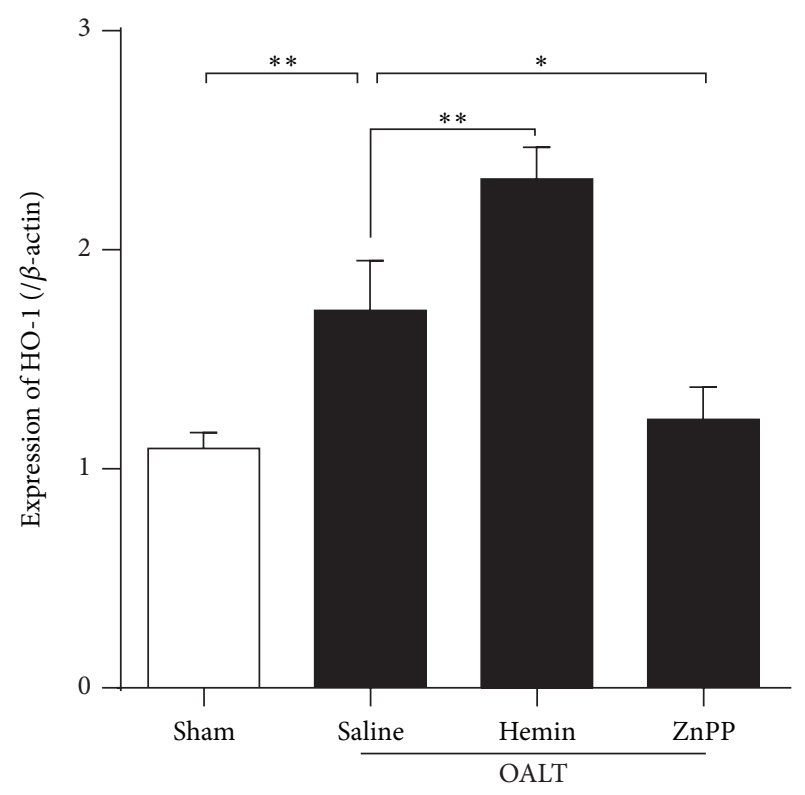

(b)

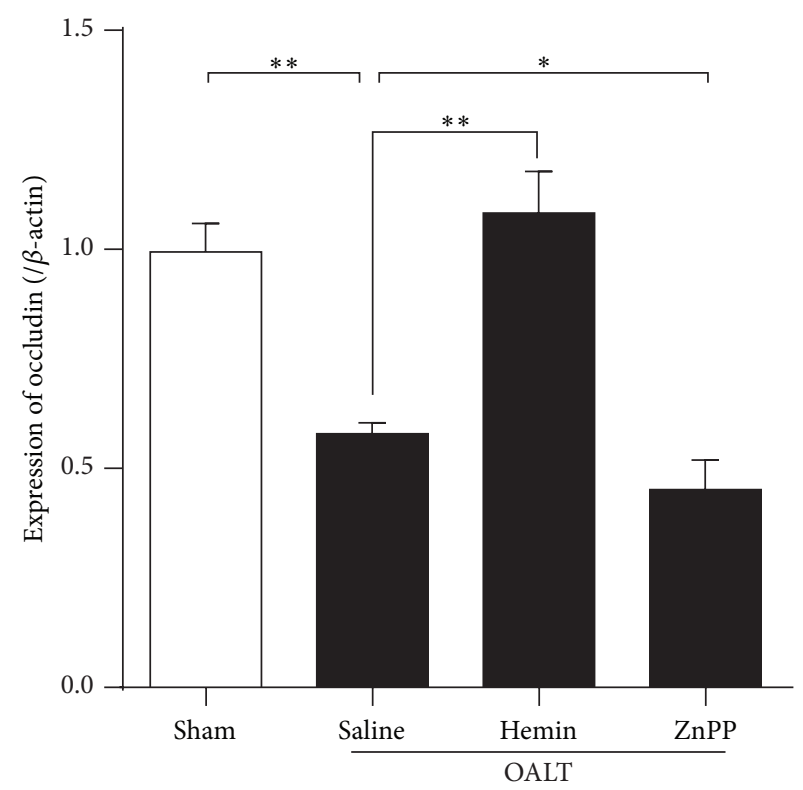

(d)

FIGURE 4: Elevated expression of HO-1 was related to the restoration of intestinal tight junction. Representative Western blots analysis (a) of the expression of HO-1 (b), ZO-1 (c), and occludin (d) in intestinal mucosa collected at $8 \mathrm{~h}$ after reperfusion and from each of 5 groups. $\beta$-actin was included as an internal standard for each blot. The results were expressed as the mean $\pm \mathrm{SD}, n=3-6 /$ group. ${ }^{*} p<0.05,{ }^{* *} p<0.01$, one-way ANOVA with Tukey test.

dysfunction. Furthermore, using HO-1 inhibitor ZnPP and agonist hemin pretreatment, we provided the evidence that increased expression of HO-1 leads to protection of TJs against disrupting intestinal epithelium. This observation is consistent with the finding that elevating HO-1 expression can facilitate the synthesis of TJ proteins in the epithelial/endothelial cell, preventing TJs from disruption [41].
Moreover, OALT elevated intestine oxidative stress level led to production of higher level MDA that could increase paracellular permeability [42]. TNF- $\alpha$, an inflammatory cytokine, which was subjected to a qualitative change observed in the TJ and redistribution of ZO-1 [43], was significantly decreased by the intervention with hemin compared with saline in rats receiving OALT in our study. Therefore, 
DAPI
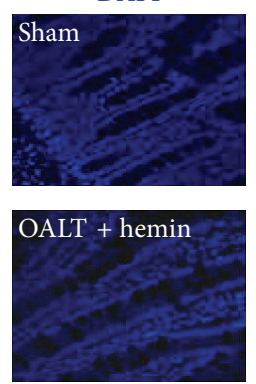

$\operatorname{Nrf} 2$
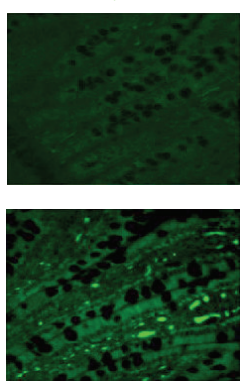

Merge
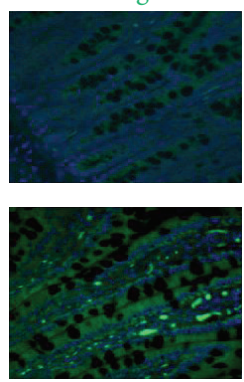

DAPI
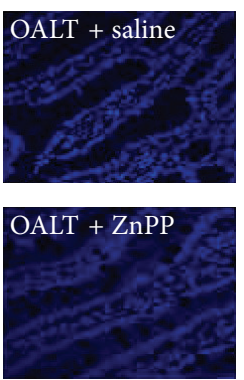

$\mathrm{Nrf2}$
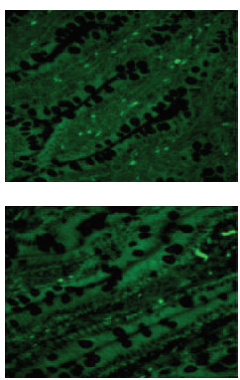

Merge
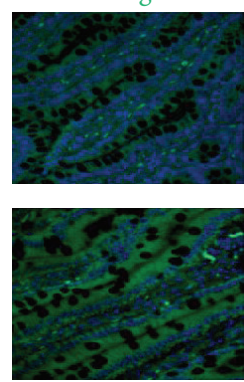

(a)

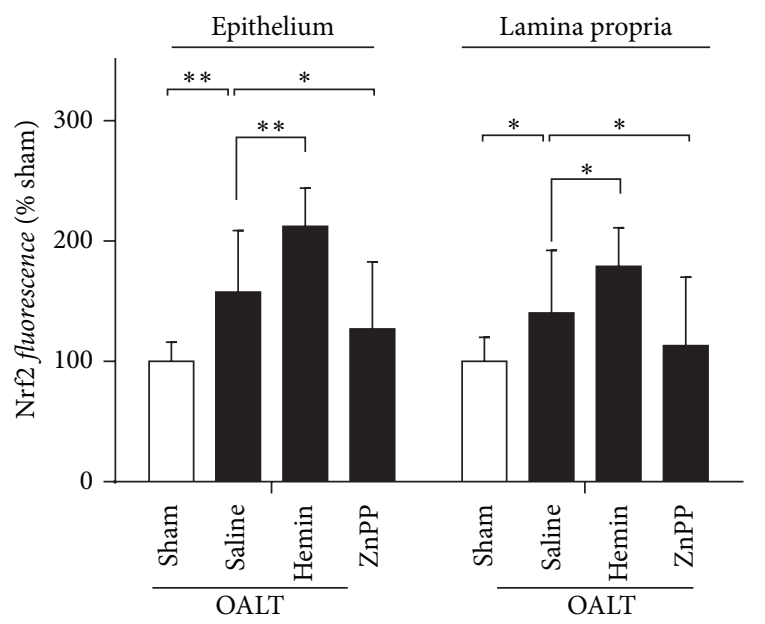

(b)

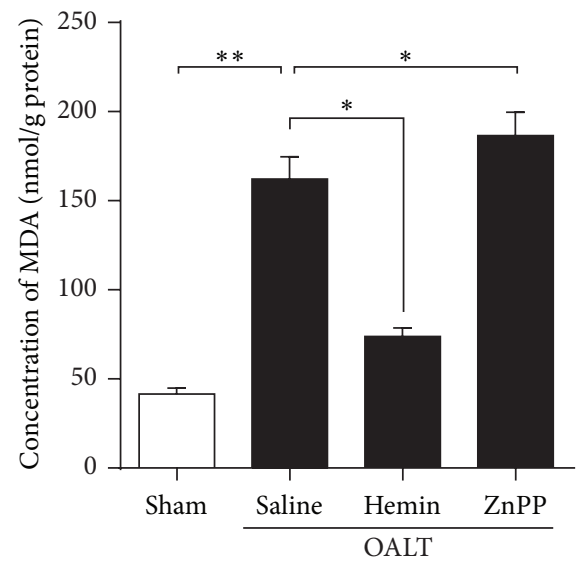

(c)

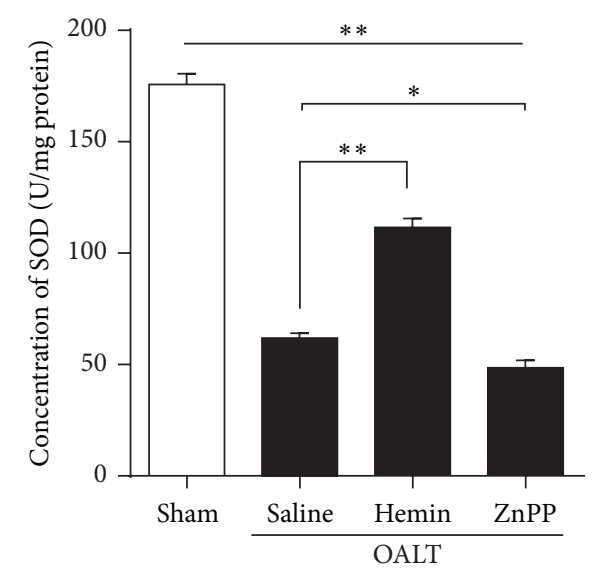

(d)

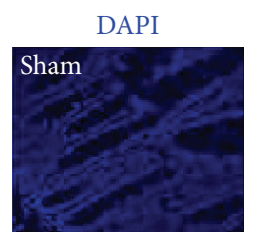

NF- «B P65

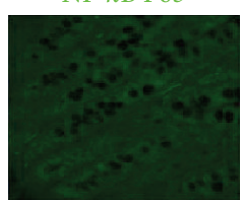

Merge
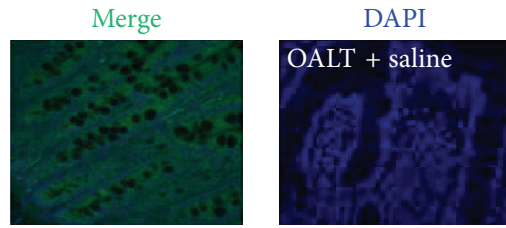

NF- $\kappa$ B P 65
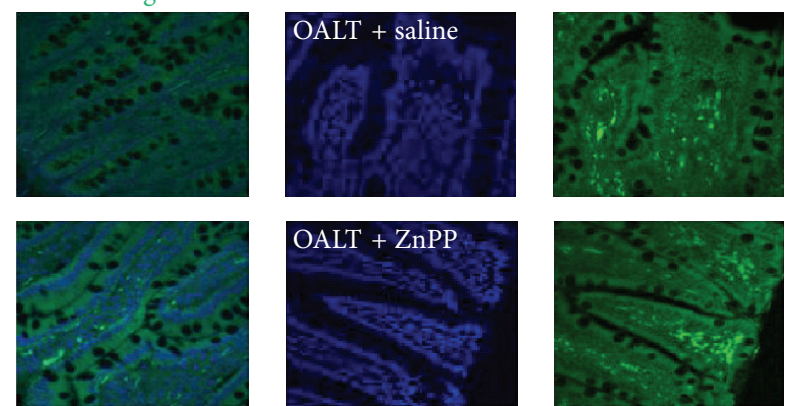

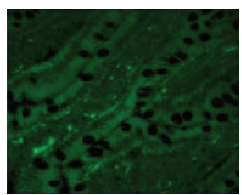

FIgUre 5: Continued. 


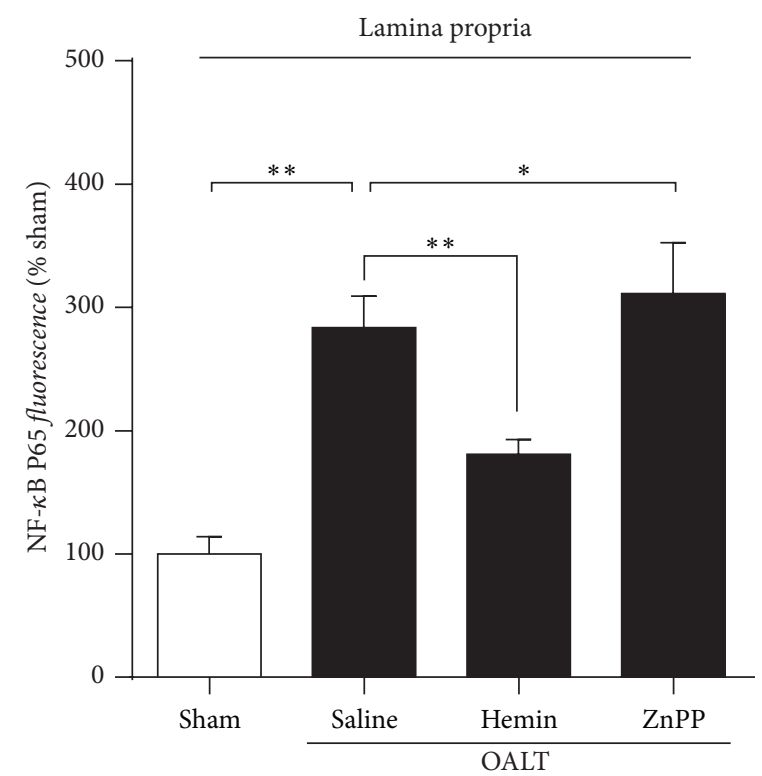

(f)

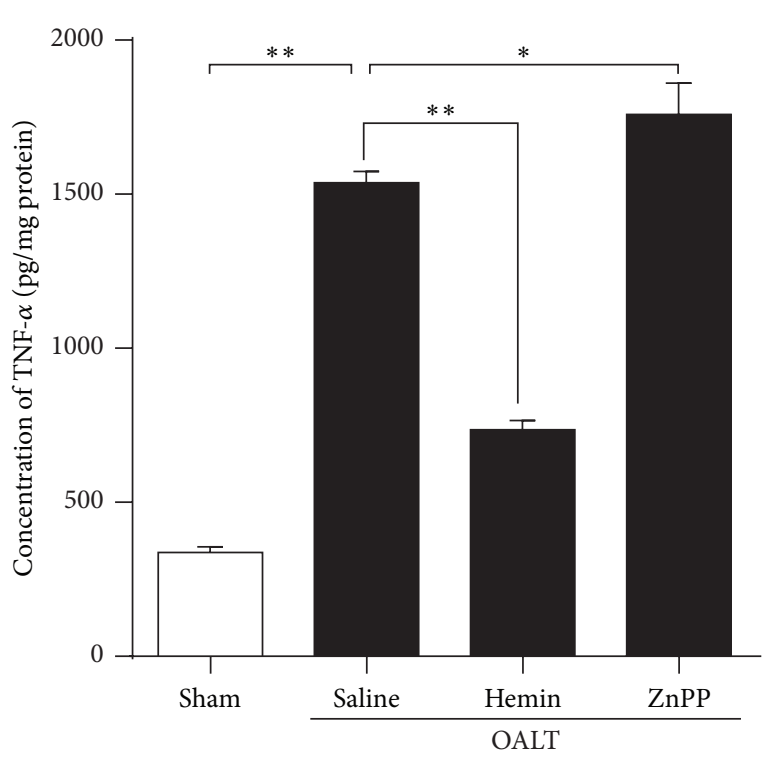

$(\mathrm{g})$

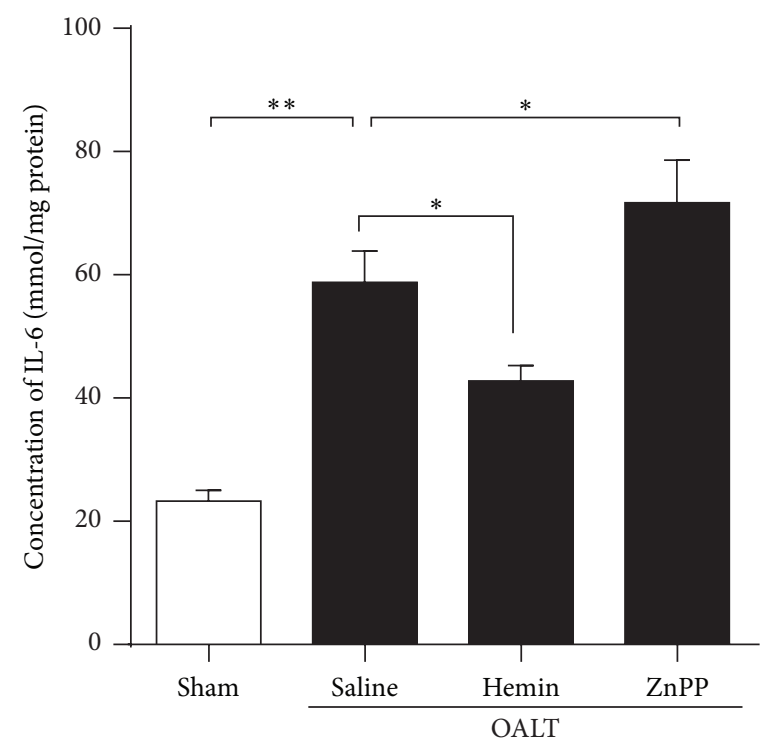

(h)

FIGURE 5: The HO-1 protective function and the translocation of transcription factors NF- $\kappa$ B and Nrf2. Immunofluorescent staining of Nrf2 (a) and NF- $\kappa \mathrm{B}$ (e) using intestinal sections harvested at $8 \mathrm{~h}$ after reperfusion from 5 groups. And fluorescence quantitation of Nrf2 in intestine epithelium and lamina propria (b) and NF- $\kappa$ B in lamina propria (f) was carried out. Malonaldehyde (MDA) (c), the end product of lipid peroxidation, was detected by TBARs method from the intestinal mucosa. The activity of superoxide dismutase (SOD) (d) that acts as an important antioxidant defense in intestine cells exposed to reactive oxygen species (ROS) was also detected by ELISA assay. The NF- $\kappa \mathrm{B}$ downstream inflammation cytokines TNF- $\alpha(\mathrm{g})$ and IL-6 (h) were also detected by ELISA assay. $\beta$-actin was included in parallel as loading reference. The results were expressed as the mean $\pm \mathrm{SD}, n=3-6 /$ group. ${ }^{*} p<0.05,{ }^{* *} p<0.01$, one-way ANOVA with Tukey test.

we tested the underlying signal pathway for HO-1 activation. We found that HO-1 activation was accompanied by the reduction of lipid peroxidation product MDA and TNF- $\alpha$ and IL- 6 cytokines, which reduced the inflammatory reaction and mitigated the damage to OALT-induced intestinal barrier.

HO-1 activation has its ability to scavenge intracellular ROS and contributes to reduction of oxidative stress. Pretreatment with HO-1 agonist hemin reduced the
OALT-initiated ROS production and barrier disorder and elevated the expression of $\mathrm{ZO}-1$ and occludin. The protective benefit of elevated HO-1 expression was also associated with more effective Nrf2 translocation, which functions as a main molecule defending the cytotoxicity of oxidative stress [44]. Nrf2 translocation led to expression of downstream antioxidant enzymes including HO-1. In this study a more frequent Nrf2 nuclear translocation in mucosa was detected 


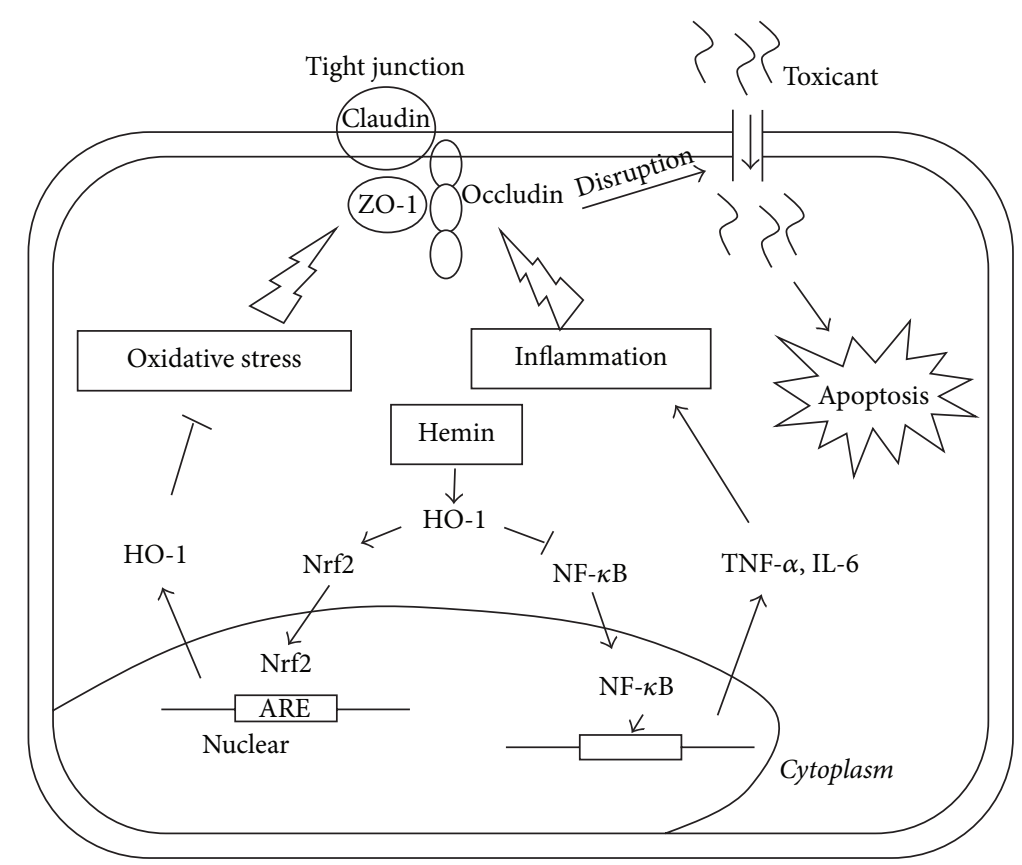

FIGURE 6: An illustration of HO-1 protection of intestinal barrier function. Intestinal inflammation and oxidative stress were induced by OALT, leading to the dysfunction of intestinal barrier that is primarily composed of tight junction (TJ). Elevation of HO-1 expression by hemin pretreatment could restore the TJ function to prevent the external toxicant from entering into cells. Cytotoxicity may cause cell apoptosis. These protective effects were associated with reduction of intercellular oxidative stress and tissue inflammation via activation of Nrf2/ARE pathway and inhibition of NF- $\kappa$ B pathway.

in hemin pretreatment group than that in control OALT group, accompanied by increased SOD activity and decreased NF- $\kappa \mathrm{B}$ translocation, which upregulates IL-6 and TNF- $\alpha$ expression. Taken together, these findings suggest that the oxidative stress likely mediated the barrier dysfunction under OALT circumstances. Our findings are consistent with published data that antioxidants can inhibit $\mathrm{H}_{2} \mathrm{O}_{2}$ induced paracellular hyperpermeability [45]. Meanwhile, the reduced TJ protein expression has been proved to play an important role in intestinal permeability in our study, which was consistent with others' study on intestine disease [46, 47]. However, as tight junction localization alteration might be more direct in studying the tight junction function [48], in vitro study will be carried out to explore the influence of inflammation and oxidative stress on tight junction localization and its underling mechanisms by using intestinal epithelium cell culture. On the other hand, although we found that HO-1 activation mitigated OALTinduced intestine injury, probably via restoring efficient expression of TJ proteins, additional studies investigating the interaction between $\mathrm{HO}-1$ and TJ proteins are warranted. As hemin was only administrated before ischemia, it is interesting to investigate impacts of hemin treatment initiated after the onset of ischemia or during reperfusion.

\section{Conclusions}

Our study showed that intestinal barrier injury and dysfunction were triggered by OALT. Furthermore, our study provided the evidence that elevated expression of
HO-1 in intestine can improve the pathological outcomes of OALT-related oxidative stress and ischemia on mucosal barrier structure and function (Figure 6). Our results offered new insights into protective function of $\mathrm{HO}-1$ in liver transplantation-induced intestinal injury. In addition, the involvement of inflammation and oxidative stress indicates that anti-inflammatory agents and antioxidants may relieve adverse effects from liver transplantation on the small intestine.

\section{Conflict of Interests}

The authors declare that they have no competing interests.

\section{Authors' Contributions}

Xinjin Chi and Weifeng Yao contributed equally to this study. Xinjin Chi and Ziqing Hei conceived and designed the experiments. Weifeng Yao and Hua Xia performed the experiments. Yi Jin and $\mathrm{Xi} \mathrm{Li}$ analyzed the data. Jun Cai contributed reagents/materials/analysis tools. Weifeng Yao wrote the paper. All authors read and approved the paper.

\section{Acknowledgments}

The study was partly supported by the Natural Science Foundation of China (no. 81471892), the Natural Science Foundation of Guangdong Province, China (no. 
S2012010008930), the Science and Technology Project Foundation of Guangdong Province, China (no. 2008B030301053; no. 2013B021800181; no. 2012B061700071), and the Outstanding Young Teacher Training Program of Sun Yat-Sen University, China (no. 14ykpy24).

\section{References}

[1] F. Åberg, H. Isoniemi, and K. Höckerstedt, "Long-Term results of liver transplantation," Scandinavian Journal of Surgery, vol. 100, no. 1, pp. 14-21, 2011.

[2] S. G. Hübscher, "What is the long-term outcome of the liver allograft?” Journal of Hepatology, vol. 55, no. 3, pp. 702-717, 2011.

[3] R. H. Wiesner and K. V. Narayanan Menon, "Late hepatic allograft dysfunction," Liver Transplantation, vol. 7, no. 11, supplement 1, pp. S60-S73, 2001.

[4] C. E. Oberkofler, P. Dutkowski, R. Stocker et al., "Model of end stage liver disease (MELD) score greater than 23 predicts length of stay in the ICU but not mortality in liver transplant recipients," Critical Care, vol. 14, no. 3, article R117, 2010.

[5] W. Yao, G. Luo, G. Zhu et al., "Propofol activation of the Nrf2 pathway is associated with amelioration of acute lung injury in a rat liver transplantation model," Oxidative Medicine and Cellular Longevity, vol. 2014, Article ID 258567, 9 pages, 2014.

[6] J. Suliburk, K. Helmer, F. Moore, and D. Mercer, "The gut in systemic inflammatory response syndrome and sepsis: enzyme systems fighting multiple organ failure," European Surgical Research, vol. 40, no. 2, pp. 184-189, 2008.

[7] Z. Urban, V. Hucthagowder, N. Schürmann et al., "Mutations in LTBP4 cause a syndrome of impaired pulmonary, gastrointestinal, genitourinary, musculoskeletal, and dermal development," The American Journal of Human Genetics, vol. 85, no. 5, pp. 593605, 2009.

[8] T. Sakai, T. Matsusaki, J. W. Marsh, I. A. Hilmi, and R. M. Planinsic, "Comparison of surgical methods in liver transplantation: retrohepatic caval resection with venovenous bypass (VVB) versus piggyback (PB) with VVB versus $\mathrm{PB}$ without VVB," Transplant International, vol. 23, no. 12, pp. 1247-1258, 2010.

[9] F. Puglisi, L. Lacitignola, A. Loverre et al., "Activation of PI3-kinase/Akt induced small bowel cell apoptosis during laparoscopic ischaemia-reperfusion of swine jejunum," Acta Chirurgica Belgica, vol. 109, no. 2, pp. 216-223, 2009.

[10] J. R. Turner, "Intestinal mucosal barrier function in health and disease," Nature Reviews Immunology, vol. 9, no. 11, pp. 799-809, 2009.

[11] D. R. Clayburgh, L. Shen, and J. R. Turner, "A porous defense: the leaky epithelial barrier in intestinal disease," Laboratory Investigation, vol. 84, no. 3, pp. 282-291, 2004.

[12] A. Farhadi, A. Banan, J. Fields, and A. Keshavarzian, "Intestinal barrier: an interface between health and disease," Journal of Gastroenterology and Hepatology, vol. 18, no. 5, pp. 479-497, 2003.

[13] H.-T. Chung, S. W. Ryter, and H. P. Kim, "Heme oxygenase-1 as a novel metabolic player," Oxidative Medicine and Cellular Longevity, vol. 2013, Article ID 814058, 2 pages, 2013.

[14] M. D. Maines, "The heme oxygenase system: a regulator of second messenger gases," Annual Review of Pharmacology and Toxicology, vol. 37, pp. 517-554, 1997.

[15] M. Coeffier, F. Le Pessot, A. Leplingard et al., "Acute enteral glutamine infusion enhances heme oxygenase-1 expression in human duodenal mucosa," The Journal of Nutrition, vol. 132, no. 9, pp. 2570-2573, 2002.

[16] S. G. R. G. Barton, D. S. Rampton, V. R. Winrow, P. Domizio, and R. M. Feakins, "Expression of heat shock protein 32 (hemoxygenase-1) in the normal and inflamed human stomach and colon: an immunohistochemical study," Cell Stress o Chaperones, vol. 8, no. 4, pp. 329-334, 2003.

[17] W. Fan, F. Huang, X. Zhu, D. Li, S. Fu, and H. He, "The heme oxygenase system and oral diseases," Oral Diseases, vol. 17, no. 3, pp. 252-257, 2011.

[18] X. Zhu, W.-G. Fan, D.-P. Li, H. Kung, and M. C. M. Lin, "Heme oxygenase-1 system and gastrointestinal inflammation: a short review," World Journal of Gastroenterology, vol. 17, no. 38, pp. 4283-4288, 2011.

[19] X. Guo, V. Y. Shin, and C. H. Cho, "Modulation of heme oxygenase in tissue injury and its implication in protection against gastrointestinal diseases," Life Sciences, vol. 69, no. 2526, pp. 3113-3119, 2001.

[20] K. J. Yun, S. C. Choi, and J. M. Oh, "Expression of heme oxygenase-1 in ischemic colitis," The Korean Journal of Gastroenterology, vol. 45, no. 5, pp. 335-339, 2005.

[21] M. J. Murray, J. J. Barbose, and C. F. Cobb, "Serum D(-)-lactate levels as a predictor of acute intestinal ischemia in a rat model," The Journal of Surgical Research, vol. 54, no. 5, pp. 507-509, 1993.

[22] M. J. Murray, M. D. Gonze, L. R. Nowak, and C. F. Cobb, “Serum $\mathrm{D}(-)$-lactate levels as an aid to diagnosing acute intestinal ischemia," The American Journal of Surgery, vol. 167, no. 6, pp. 575-578, 1994.

[23] S. M. Smith, R. H. K. Eng, and F. Buccini, "Use of D-lactic acid measurements in the diagnosis of bacterial infections," The Journal of Infectious Diseases, vol. 154, no. 4, pp. 658-664, 1986.

[24] H. Funaoka, T. Kanda, S. Kajiura, Y. Ohkaru, and H. Fujii, "Development of a high-specificity sandwich ELISA system for the quantification of human intestinal fatty acid-binding protein (I-FABP) concentrations," Immunological Investigations, vol. 40, no. 3, pp. 223-242, 2011.

[25] L. Shen, C. R. Weber, D. R. Raleigh, D. Yu, and J. R. Turner, "Tight junction pore and leak pathways: a dynamic duo," Annual Review of Physiology, vol. 73, pp. 283-309, 2011.

[26] C. R. Weber, "Dynamic properties of the tight junction barrier," Annals of the New York Academy of Sciences, vol. 1257, no. 1, pp. 77-84, 2012.

[27] J. M. Anderson and C. M. van Itallie, "Tight junctions and the molecular basis for regulation of paracellular permeability," The American Journal of Physiology, vol. 269, no. 4, part 1, pp. G467G475, 1995.

[28] I. Martìn-Padura, S. Lostaglio, M. Schneemann et al., "Junctional adhesion molecule, a novel member of the immunoglobulin superfamily that distributes at intercellular junctions and modulates monocyte transmigration," The Journal of Cell Biology, vol. 142, no. 1, pp. 117-127, 1998.

[29] S. Tsukita and M. Furuse, "Occludin and claudins in tightjunction strands: leading or supporting players?" Trends in Cell Biology, vol. 9, no. 7, pp. 268-273, 1999.

[30] E. G. Laws and D. E. Freeman, "Effects of heparin, venous strangulation obstruction of the small intestine, and reperfusion of the small intestine on plasma diamine oxidase activity in horses," American Journal of Veterinary Research, vol. 55, no. 2, pp. 185-191, 1994.

[31] X. Chi, A. Zhang, G. Luo et al., "Knockdown of myeloid differentiation protein-2 reduces acute lung injury following 
orthotopic autologous liver transplantation in a rat model," Pulmonary Pharmacology \& Therapeutics, vol. 26, no. 3, pp. 380-387, 2013.

[32] C. Luo, D. Yuan, X. Li et al., "Propofol attenuated acute kidney injury after orthotopic liver transplantation via inhibiting gap junction composed of connexin 32," Anesthesiology, vol. 122, no. 1, pp. 72-86, 2015.

[33] W. Zhao, S. Zhou, W. Yao et al., "Propofol prevents lung injury after intestinal ischemia-reperfusion by inhibiting the interaction between mast cell activation and oxidative stress," Life Sciences, vol. 108, 80, no. 2, p. 87, 2014.

[34] L. C. J. M. Lemaire, B. A. van Wagensveld, T. M. van Gulik, J. Dankert, J. J. B. van Lanschot, and D. J. Gouma, "Bacterial translocation to the thoracic duct in a setting of ischemia, partial resection and reperfusion of the porcine liver," Digestive Surgery, vol. 16, no. 3, pp. 222-228, 1999.

[35] E. Abdala, C. E. S. Baía, S. Mies et al., "Bacterial translocation during liver transplantation: a randomized trial comparing conventional with venovenous bypass vs. piggyback methods," Liver Transplantation, vol. 13, no. 4, pp. 488-496, 2007.

[36] K. S. Filos, I. Kirkilesis, I. Spiliopoulou et al., "Bacterial translocation, endotoxaemia and apoptosis following Pringle manoeuvre in rats," Injury, vol. 35, no. 1, pp. 35-43, 2004.

[37] J.-H. Yao, X.-S. Zhang, S.-S. Zheng et al., "Prophylaxis with carnosol attenuates liver injury induced by intestinal ischemia/reperfusion," World Journal of Gastroenterology, vol. 15, no. 26, pp. 3240-3245, 2009.

[38] C. Hualin, X. Wenli, L. Dapeng, L. Xijing, P. Xiuhua, and P. Qingfeng, "The anti-inflammatory mechanism of heme oxygenase-1 induced by hemin in primary rat alveolar macrophages," Inflammation, vol. 35, no. 3, pp. 1087-1093, 2012.

[39] G. Sass, M. C. P. Soares, K. Yamashita et al., "Heme oxygenase-1 and its reaction product, carbon monoxide, prevent inflammation-related apoptotic liver damage in mice," Hepatology, vol. 38, no. 4, pp. 909-918, 2003.

[40] K. L. Edelblum and J. R. Turner, "The tight junction in inflammatory disease: communication breakdown," Current Opinion in Pharmacology, vol. 9, no. 6, pp. 715-720, 2009.

[41] W. Xie, H. Wang, L. Wang, C. Yao, R. Yuan, and Q. Wu, "Resolvin D1 reduces deterioration of tight junction proteins by upregulating HO-1 in LPS-induced mice," Laboratory Investigation, vol. 93, no. 9, pp. 991-1000, 2013.

[42] T. Maeda, Y. Miyazono, K. Ito, K. Hamada, S. Sekine, and T. Horie, "Oxidative stress and enhanced paracellular permeability in the small intestine of methotrexate-treated rats," Cancer Chemotherapy and Pharmacology, vol. 65, no. 6, pp. 1117-1123, 2010.

[43] L. S. Poritz, K. I. Garver, A. F. Tilberg, and W. A. Koltun, "Tumor necrosis factor alpha disrupts tight junction assembly," The Journal of Surgical Research, vol. 116, no. 1, pp. 14-18, 2004.

[44] R. Gold, L. Kappos, D. L. Arnold et al., "Placebo-controlled phase 3 study of oral BG-12 for relapsing multiple sclerosis," The New England Journal of Medicine, vol. 367, no. 12, pp. 1098-1107, 2012.

[45] R. K. Rao, L. Li, R. D. Baker, S. S. Baker, and A. Gupta, "Glutathione oxidation and PTPase inhibition by hydrogen peroxide in Caco-2 cell monolayer," American Journal of PhysiologyGastrointestinal and Liver Physiology, vol. 279, no. 2, pp. G332G340, 2000.

[46] B. Chang, L. Sang, Y. wang, J. Tong, D. Zhang, and B. Wang, "The protective effect of VSL\#3 on intestinal permeability in a rat model of alcoholic intestinal injury," BMC Gastroenterology, vol. 13, no. 1, article 151, 2013.

[47] A. Casselbrant, E. Elias, L. Fändriks, and V. Wallenius, "Expression of tight-junction proteins in human proximal small intestinal mucosa before and after Roux-en-Y gastric bypass surgery," Surgery for Obesity and Related Diseases, vol. 11, no. 1, pp. 45-53, 2015.

[48] R. Ciccocioppo, A. Finamore, C. Ara, A. Di Sabatino, E. Mengheri, and G. R. Corazza, "Altered expression, localization, and phosphorylation of epithelial junctional proteins in celiac disease," American Journal of Clinical Pathology, vol. 125, no. 4, pp. 502-511, 2006. 


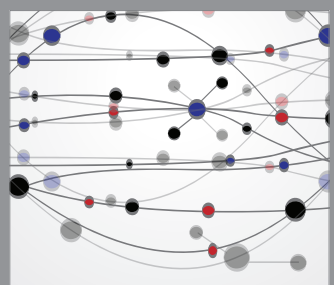

The Scientific World Journal
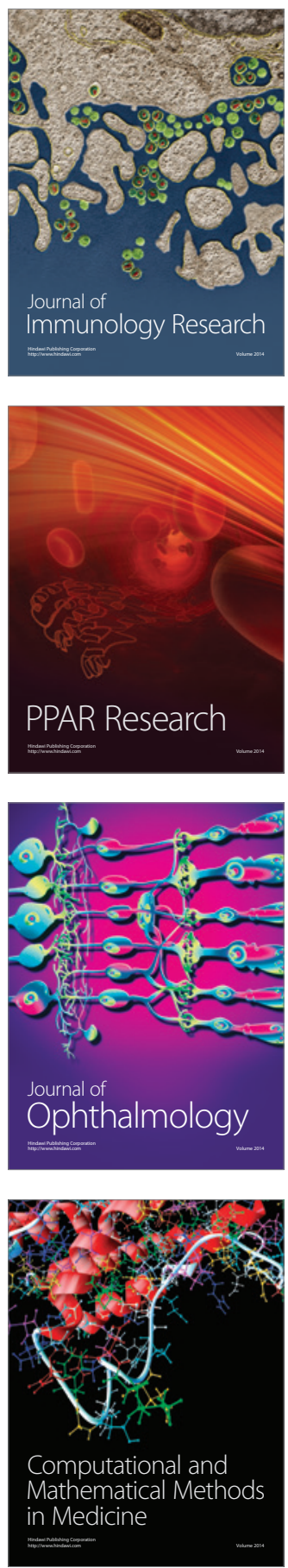

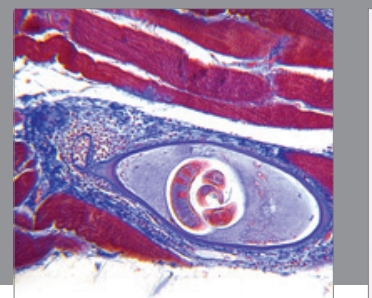

Gastroenterology

Research and Practice
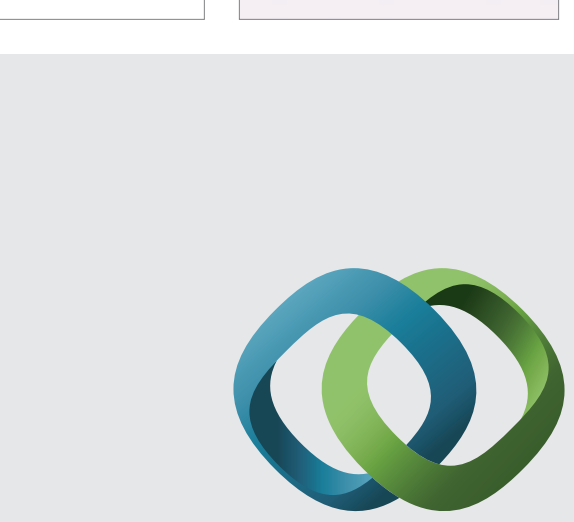

\section{Hindawi}

Submit your manuscripts at

http://www.hindawi.com
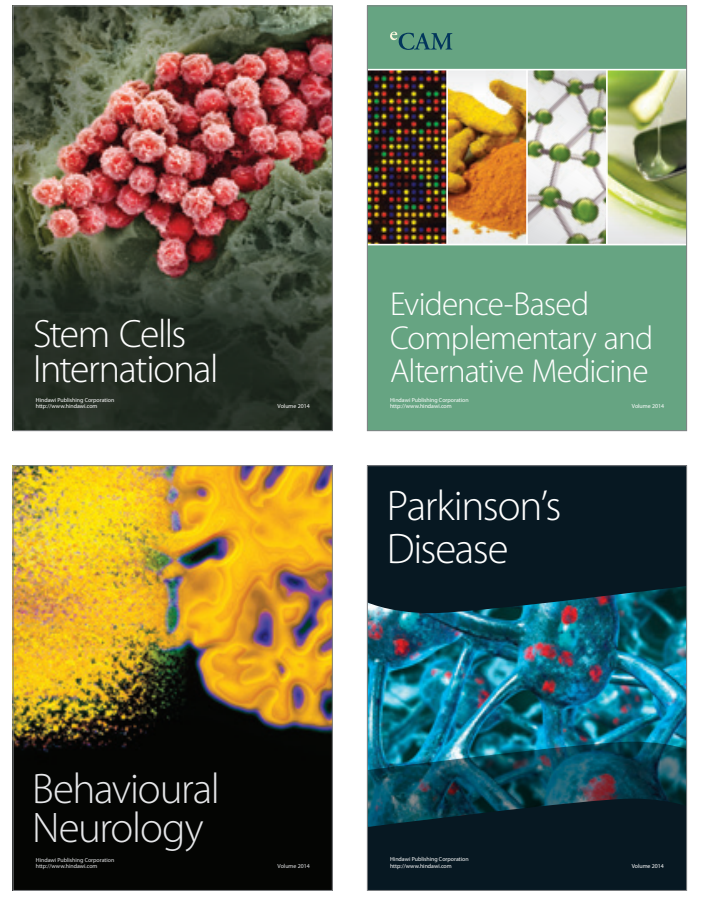
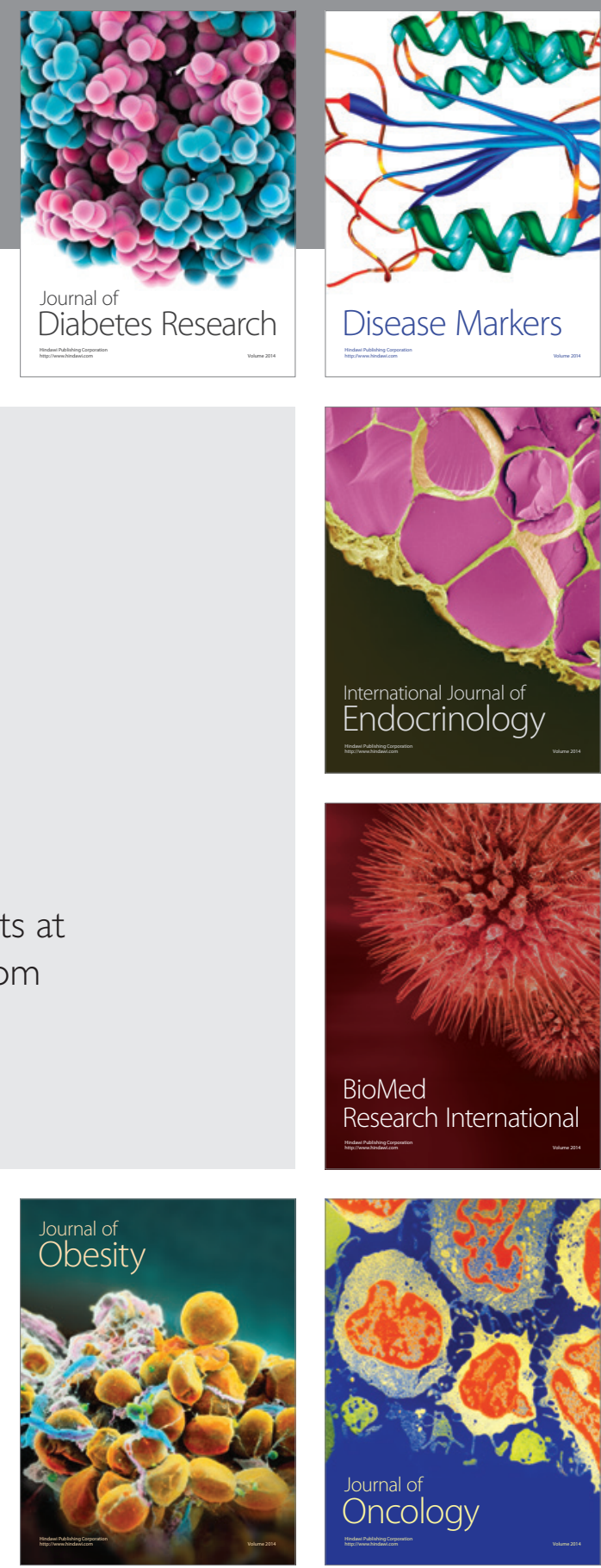

Disease Markers
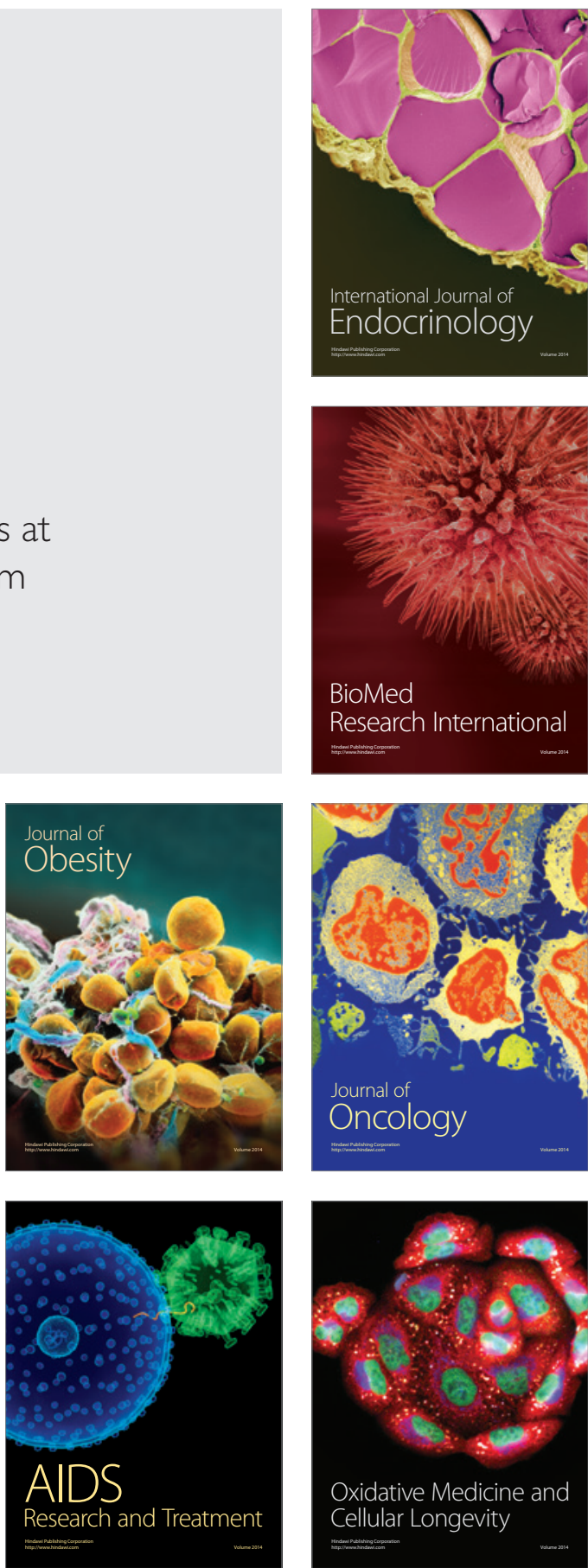